\title{
Planejamento em saúde sobre práticas corporais e atividade física no Programa Academia da Saúde
}

\section{Health planning on body practices and physical activity in the health academy program}

\begin{abstract}
AUTORES
Sofia Wolker Manta ${ }^{1}$ (D)

Paula Fabricio Sandreschi ${ }^{1}$ (D)

Emanuele Naiara Quadros ${ }^{1}$ iD

Paulo Vitor de Souza ${ }^{1}$ (D)

Cassiano Ricardo $\operatorname{Rech}^{1}$ (D)

Tânia Rosane Bertoldo Benedetti ${ }^{1}$ (D)

1 Universidade Federal de Santa Catarina,

Departamento de Educação Física, Florianópolis,

Santa Catarina, Brasil.

\section{CONTATO}

Sofia Wolker Manta

sofiawolker@gmail.com

SHS quadra 6, conjunto $A$, sem número.

Bairro: Asa Sul, Brasília, Distrito Federal.

CEP: 70316-000.

DOI
\end{abstract}

$10.12820 /$ rbafs. $25 \mathrm{e} 0168$

\begin{abstract}
RESUMO
O objetivo foi analisar o planejamento das práticas corporais e atividades físicas (PC/AF) dos profissionais de educação física concluintes do Curso de Aperfeiçoamento em Implementação da Política Nacional de Promoção da Saúde: Programa Academia da Saúde (PAS). Das ações analisadas ( $\mathrm{n}=$ $149), 60,5 \%(n=286)$ eram de PC/AF. A ginástica foi mais frequente $(20,1 \% ; n=95)$ e $21,4 \%(n=$ 101) das práticas tinham a alimentação associada. Mais de $60 \%$ das ações não informaram instrumentos $(n=330)$, cronograma $(n=345)$, recursos financeiros $(n=366)$ e alcance dos usuários $(n=$ 394). A atuação multiprofissional foi prevista em $61,7 \%(n=292)$ das ações. Sendo assim, o planejamento em saúde carece de informações metodológicas, avaliação e público-alvo para implementação de ações.
\end{abstract}

Palavras-chave: Atividade motora; Saúde pública; Planejamento; Saúde.

ABSTRACT

The aim was to analyze the planning of body practices and physical activities (BP/PA) of physical education professionals concluding the Improvement Course in Implementation of the National Health Promotion Policy: Academia da Saude Program (PAS). Of the analyzed actions $(n=149), 60.5 \%(n=286)$ were of BP/PA. Gymnastics was more frequent $(20.1 \% ; n=95)$ and $21.4 \%(n=101)$ of the practices had associated food. More than $60 \%$ of the actions did not inform instruments $(n=330)$, schedule $(n=345)$, financial resources $(n=366)$ and reach of users $(n=394)$. Multiprofessional action was expected in $61.7 \%(n=292)$ of the actions. Thus, health planning lacks methodological information, assessment, and target audience for implementing actions.

Keywords: Motor activity; Public health; Planning; Health.

\section{(cc) BY}

Este trabalho está licenciado com uma Licença

Creative Commons - Atribuição 4.0 Internacional.

\section{Introdução}

A reformulação da Política Nacional de Promoção de Saúde (PNPS) trouxe incentivos para a promoção de ações em práticas corporais e atividades físicas (PC/ AF) no contexto do Sistema Único de Saúde (SUS) no Brasil $^{1}$. O documento prevê como meta o aumento de até $10 \%$ na proporção de pessoas fisicamente ativas e redução do comportamento sedentário ${ }^{2}$.

Dessa forma, as ações de PC/AF são incentivadas na Atenção Primária à Saúde (APS) ${ }^{1}$. Desde 2011, o Programa Academia da Saúde (PAS) está entre as principais estratégias de promoção e produção do cuidado à saúde, por meio da implantação de polos que contam com infraestrutura, equipamentos e profissionais qualificados no território brasileiro ${ }^{4}$. Ainda, no período de 2015 a 2017 foi observado um crescimento no número de polos em funcionamento e nas ações de $\mathrm{PC} / \mathrm{AF}^{4}$, o que contribuiu para o fortalecimento das redes da APS em ações intersetoriais e multidisciplinares. Além disso, os profissionais de educação física estão entre aqueles que mais registram ações em PC/ AF nos polos do programa ${ }^{4,5}$.

O planejamento em saúde, para o desenvolvimento de ações de $\mathrm{PC} / \mathrm{AF}$, é essencial para aumentar a autonomia e engajamento dos usuários ${ }^{6-8}$. Para isso, é necessário fornecer o suporte aos profissionais de saúde, para que sejam desenvolvidas ações efetivas e sustentáveis na $\mathrm{APS}^{9-12}$. Planejar consiste em atender às características territoriais, conduzir a previsão da execução das propostas, com objetivos, metas, resultados esperados, relevância e atuação dos profissionais de saúde ${ }^{8,12}$. Por isso, analisar o planejamento de profissionais de saúde 
no PAS para a implementação de ações em PC/AF é uma forma de compreender a utilização de múltiplas estratégias, no que diz respeito à estrutura, processo e resultados $^{9-11}$. Os estudos mostram que a atuação dos profissionais de saúde na APS pouco relata sobre o planejamento em saúde e quais as estratégias adotadas para a implementação das ações ${ }^{9,10}$.

Sendo assim, o objetivo do estudo foi analisar o planejamento em saúde das ações de práticas corporais e atividades físicas dos profissionais de educação física concluintes do Curso de Aperfeiçoamento em Implementação da Política Nacional de Promoção da Saúde: Programa Academia da Saúde.

\section{Métodos}

O estudo caracteriza-se por ser descritivo do tipo documental ${ }^{13}$. O objetivo foi identificar e descrever o planejamento em saúde elaborado pelos profissionais de educação física para desenvolvimento de ações de PC/AF.

Os documentos analisados foram os trabalhos entregues pelos profissionais de educação física concluintes da sétima edição do Curso de Aperfeiçoamento em Implementação da Política Nacional de Promoção da Saúde: Programa Academia da Saúde. Este curso foi ofertado em 2017 pela Universidade Federal de Santa Catarina (UFSC) e a Fundação de Ensino e Engenharia de Santa Catarina (FEESC) em parceria com Ministério da Saúde, por meio do Departamento de Vigilância de Doenças e Agravos não Transmissíveis e Promoção da Saúde (DANTPS) da Secretaria de Vigilância em Saúde (SVS). O curso foi ofertado online, via moodle e ocorreu de 26 de junho de 2017 a 21 de janeiro de 2018, com uma carga-horária de 180 horas $^{12}$.

A orientação aos profissionais do curso para a entrega das propostas era elaborar um planejamento contendo alguma ação em promoção de saúde para ser implementado no contexto da APS. A proposta deveria refletir a realidade do local de trabalho dos profissionais, sendo sintetizada de forma objetiva, coerente e clara, com levantamento sobre a situação de saúde do município, para assim garantir a execução ${ }^{12}$.

Para a análise documental, as informações foram extraídas dos trabalhos e categorizadas ${ }^{13}$ quanto a presença ou não da informação solicitada. A análise dos documentos foi realizada em etapas:

a) Seleção e identificação documental das propostas elaboradas pelos profissionais de educação física: um dos autores (SWM) fez a solicitação dos trabalhos e identificou aqueles que atendiam aos critérios de inclusão (ter sido delineada por um profissional de educação física);

b) Análise documental por pares: dois autores (ENQe PVS) realizaram a leitura sucessiva e na íntegra dos documentos, para posterior categorização e tabulação das informações;

c) Categorização das informações: dois autores (ENQ e PVS), independentemente, após a leitura documental das propostas, criaram categorias de análise para a extração das informações. As categorias foram criadas com base nos seguintes temas: (1) Descrição da ação proposta (exemplos: tipo da ação; frequência, duração semanal e local onde será desenvolvida); (2) A atividade física como uma ação prevista; (3) Ações combinadas à atividade física; (4) Metodologia adotada para o desenvolvimento da ação; (5) Modalidade de atividade física desenvolvida; (6) Uso de instrumentos para avaliar a atividade física; (7) Ação colaborativa com outros profissionais de saúde do local de trabalho; (8) Cronograma de desenvolvimento da ação; (9) É apresentado a previsão de gastos com recursos financeiros para o desenvolvimento da ação; (10) Público-alvo atendido com a ação; (11) Atendimento às populações especiais; (12) Identificação da população especial atendida; e, (13) Previsão sobre a quantidade de pessoas atendidas pela ação.

d) Extração das informações: dois autores (ENQ e PVS), independentemente, realizaram a extração e tabulação das informações com base nas categorias. Para isso, as informações foram organizadas em planilha do excel contendo informações dos proponentes das ações, sexo, atuação e formação profissional. Além dessas informações as informações tabuladas foram codificadas em dicotômicas ou politômicas;

e) Controle de qualidade da extração: após a extração das informações foi realizado o controle de qualidade (concordância das informações extraídas) em $10 \%$ de cada planilha preenchida por um dos autores (SWM).

As informações tabuladas foram convertidas para o pacote IMB SPSS ${ }^{\circledR}$ versão 21 . Empregou-se a frequência absoluta e relativa para descrição das variáveis. Todos os procedimentos do estudo foram aprovados no Comitê de Ética em Pesquisa da Universidade Federal de Santa Catarina sob número de parecer 2.252.884 e CAAE 67635917.3.0000.0121. 


\section{Resultados}

Foram identificadas 155 propostas de profissionais de Educação Física, porém seis foram excluídas por não reportarem ações em planejamento e sim ações já em desenvolvimento nos seus municípios. Assim, a análise documental final foi de 149 propostas.

Com relação ao perfil sociodemográfico dos profissionais de Educação Física que elaboraram as propostas, $55,7 \%(\mathrm{n}=83)$ eram do sexo feminino. A maior proporção dos profissionais tinha especialização $(43,0 \%)$ e atuava no Programa Academia da Saúde $(53,7 \%)$ - Tabela 1.

Tabela 1 - Perfil dos profissionais de Educação Física que enviaram propostas de implementação de ações em atividades físicas no Programa Academia da Saúde $(\mathrm{n}=149)$.

\begin{tabular}{lcccccc}
\hline \multirow{2}{*}{ Descrição profissionais } & \multicolumn{3}{c}{ Total } & \multicolumn{2}{c}{$\begin{array}{c}\text { Masculino } \\
(\mathrm{n}=66)\end{array}$} & \multicolumn{2}{c}{$\begin{array}{c}\text { Feminino } \\
(\mathrm{n}=83)\end{array}$} \\
\cline { 2 - 8 } & $\mathrm{n}$ & $\%$ & $\mathrm{n}$ & $\%$ & $\mathrm{n}$ & $\%$ \\
\hline Formação & 23 & 15,4 & 6 & 9,1 & 17 & 20,5 \\
Graduação & 64 & 43,0 & 19 & 28,8 & 45 & 54,2 \\
Especialização & 10 & 6,7 & 1 & 1,5 & 9 & 10,8 \\
Mestrado & 1 & 0,7 & 1 & 1,5 & 0 & 0,0 \\
Doutorado & 18 & 12,1 & 6 & 9,1 & 12 & 14,5 \\
Não responderam & & & & & & \\
Atuação profissional & 80 & 53,7 & 33 & 50,0 & 47 & 56,6 \\
Programa Academia da Saúde & 69 & 46,3 & 33 & 50,0 & 36 & 43,4 \\
Outros & & & & &
\end{tabular}

Das 149 propostas analisadas foi identificado um total de 473 ações, sendo que $286(60,5 \%)$ relatava alguma PC/AF (Tabela 2). Em 72,3\% ( $=342$ ) das propostas não eram previstas ações combinadas às $\mathrm{PC} /$ $\mathrm{AF}$ e, quando combinadas, a alimentação foi a mais frequente $(21,4 \%)$. A metodologia das ações em sua maioria era composta de grupos de atividades práticas de PC/AF (44,0\%), porém em 24,3\% não continha especificações das características metodológicas. Quanto aos tipos de atividades, a ginástica e a dança foram as mais frequentes nos planejamentos $(20,1 \%$ e $8,7 \%$, respectivamente). $60,9 \%$ das propostas não descreveram o uso de instrumentos para avaliação dos objetivos. A previsão de atuação multiprofissional foi presente em $61,7 \%$ das ações. A descrição sobre o cronograma para a execução (tempo previsto) e orçamento financeiro para o desenvolvimento da ação não foram especificados em mais de $70 \%$ das propostas. Apenas 11,2\% e 5,5\% apresentaram a população-alvo a ser atendida (crianças/adolescentes e idosos, respectivamente). Em 83,3\% dos planejamentos não foram previstos atendimentos a alguma população em condição especial, mas, quando presentes, os indivíduos obesos foram mais citados (5,1\%). Por fim, 98,7\% das propostas não mencionaram a quantidade prevista do público atendido, apesar de quatro planejamentos informarem o atendimento entre 12 a 128 pessoas (Tabela 2).

Tabela 2 - Descrição das ações propostas no planejamento das práticas corporais e atividades físicas propostas pelos profissionais de educação física ( $\mathrm{n}=473)$.

\begin{tabular}{|c|c|c|}
\hline Descrição das ações planejadas & $\mathrm{n}$ & $\%$ \\
\hline \multicolumn{3}{|l|}{ Planejamento de atividades de PC/AF } \\
\hline $\operatorname{Sim}$ & 286 & 60,5 \\
\hline Não & 187 & 39,5 \\
\hline \multicolumn{3}{|l|}{ Previsão de outras ações combinadas às PC/AF } \\
\hline Não especifica & 342 & 72,3 \\
\hline Alimentação & 101 & 21,4 \\
\hline Reabilitação & 18 & 3,8 \\
\hline PICs & 12 & 2,5 \\
\hline \multicolumn{3}{|l|}{ Metodologia adotada para desenvolver a ação de PC/AF } \\
\hline Grupos práticos de $\mathrm{PC} / \mathrm{AF}$ & 208 & 44,0 \\
\hline Palestras/oficinas & 43 & 9,1 \\
\hline Aconselhamentos & 33 & 7,0 \\
\hline Mais de uma metodologia & 32 & 6,8 \\
\hline Grupos de conversas & 29 & 6,1 \\
\hline Atendimento individual & 10 & 2,1 \\
\hline Distribuição de panfletos & 3 & 0,6 \\
\hline Não especifica & 115 & 24,3 \\
\hline \multicolumn{3}{|l|}{ Modalidade da ação prevista de PC/AF } \\
\hline Não especifica & 240 & 50,7 \\
\hline Ginásticas & 95 & 20,1 \\
\hline Danças & 41 & 8,7 \\
\hline $\begin{array}{l}\text { Atividades aeróbicas (condicionamento, fortalecimento, } \\
\text { caminhadas) }\end{array}$ & 23 & 4,9 \\
\hline Alongamentos & 22 & 4,7 \\
\hline Lutas & 19 & 4,0 \\
\hline Esportes coletivos/individuais & 18 & 3,8 \\
\hline PICs & 11 & 2,3 \\
\hline Reabilitação & 2 & 0,4 \\
\hline Ações pontuais (ex: dia da caminhada) & 2 & 0,4 \\
\hline \multicolumn{3}{|l|}{ Previsão do uso de instrumentos para avaliar as $\mathrm{PC} / \mathrm{AF}$} \\
\hline Sim & 143 & 30,2 \\
\hline Não & 330 & 69,8 \\
\hline
\end{tabular}

Continua... 


\begin{tabular}{|c|c|c|}
\hline Descrição das ações planejadas & $\mathrm{n}$ & $\%$ \\
\hline \multicolumn{3}{|c|}{ Previsão de atuação multiprofissional na ação } \\
\hline Sim & 292 & 61,7 \\
\hline Não & 181 & 38,3 \\
\hline \multicolumn{3}{|c|}{ Previsão de cronograma de execução para a ação } \\
\hline Sim & 128 & 27,1 \\
\hline Não & 345 & 72,9 \\
\hline \multicolumn{3}{|c|}{ Previsão de recursos financeiros para a execução da ação } \\
\hline Sim & 107 & 22,6 \\
\hline Não & 366 & 77,4 \\
\hline \multicolumn{3}{|c|}{ Previsão da quantidade de participantes envolvidos na ação } \\
\hline $\operatorname{Sim}$ & 6 & 1,3 \\
\hline Não & 467 & 98,7 \\
\hline \multicolumn{3}{|l|}{ Público-alvo atendido na ação } \\
\hline Não especifica & 229 & 48,4 \\
\hline Todas as faixas-etárias & 83 & 17,5 \\
\hline Crianças/adolescentes & 53 & 11,2 \\
\hline Adultos & 42 & 8,9 \\
\hline Idosos & 26 & 5,5 \\
\hline Adultos/idosos & 22 & 4,7 \\
\hline Crianças/adolescentes/adultos & 18 & 3,8 \\
\hline \multicolumn{3}{|c|}{ Ação planejada para atender a populações especiais } \\
\hline Não especifica & 394 & 83,3 \\
\hline Mais de uma população especial & 40 & 8,5 \\
\hline Obesos & 24 & 5,1 \\
\hline Hipertensos & 6 & 1,3 \\
\hline Outros & 2 & 1,1 \\
\hline Diabéticos & 3 & 0,6 \\
\hline Doenças mentais & 1 & 0,2 \\
\hline
\end{tabular}

$\mathrm{PC} / \mathrm{AF}$ = práticas corporais e atividades físicas; $\mathrm{PICs}$ = práticas integrativas complementares.

\section{Discussão}

Segundo as informações, mais de $60 \%$ dos profissionais de educação física contemplaram o desenvolvimento de alguma PC/AF e pouco mais de $20 \%$ destas, era ação combinada com alimentação. As propostas, em sua maioria, não definiram as características metodológicas, a modalidade a ser oferecida, o uso de instrumentos para a avaliação dos resultados, não especificaram o cronograma de trabalho da ação e o orçamento para o desenvolvimento. Quanto ao perfil da população a ser atendida, maior proporção não especificou a faixa etária para atendimento, e menos de um terço das propostas previu a participação de todas as faixas etárias.
Mais de $80 \%$ das propostas não foram planejadas para atender alguma necessidade de saúde da população e quase a totalidade das ações não informaram a quantidade de pessoas que poderiam ser atendidas.

O planejamento em saúde para as ações em promoção de saúde na saúde pública é um grande desafio ${ }^{8}$. O planejamento das ações na APS precisa ser factível aos contextos e eficaz para contornar os agravos à saúde, bem como manutenção dos hábitos saudáveis ${ }^{1,3,11}$. De modo geral, observou-se que os planejamentos não deixam claro a organização e estruturação do que propor, como, com quem e para quem ${ }^{9,14}$. Portanto, um planejamento para implementação na saúde pública deve analisar os recursos humanos, financeiros e de gestão e, assim intervir de forma eficaz, com baixo custo e resolubilidade ${ }^{11}$.

Nos planejamentos analisados, a ginástica e a dança foram mais presentes, o que também foi observado em outros estudos que investigaram o contexto do PAS ${ }^{5,15}$. Ademais, a atividade física e a alimentação saudável estão entre as ações mais frequentemente relatadas nos municípios brasileiros ${ }^{16}$. O presente estudo mostrou que menos de $10 \%$ das ações preveem metodologias combinadas, além da essência prática dos encontros, destacaram com frequência a atuação multiprofissional. $\mathrm{O}$ trabalho com equipe multiprofissional tem sido reportado por profissionais de educação física como um importante facilitador para trocas de informações e integralidade do cuidado, o que pode favorecer a adoção das ações pelos profissionais da saúde nos ambientes de trabalho, Além disso, isso reduz as chances de ações isoladas ou sobrepostas entre profissionais de saúde, mesmo diante distintos objetivos ${ }^{7}$. Estudos mostram que ausência de planejamento e a ineficiência dos processos de trabalho, nos serviços da rede de Atenção Primária, dificultam a articulação das atividades programadas às demais ações ${ }^{6-8}$, Possivelmente, ações planejadas com base em evidências, com participação social e da equipe de profissionais do local, contendo ambiente e equipamentos adequados, além do controle e acompanhamento dos objetivos pretendidos aumentam as chances de que estas sejam sustentáveis na APS $^{17,18}$.

Em pouco menos de $50 \%$ das ações não foi identificado o perfil de atendidos, como a faixa etária ou um diagnóstico situacional sobre problemas de saúde, o que denota possivelmente uma dificuldade no domínio dos conteúdos técnicos e teóricos para planejamentos em saúde ${ }^{10,19}$. Buscar as evidências situacionais de saúde da população local e até mesmo recomendações ou protocolos que guiam a orientação do planejamento da 
ação são importantes para o embasamento teórico de ações ${ }^{20}$. Ademais, a previsão da quantidade de pessoas alcançadas e de como acontecerão as estratégias de alcance, inclusão dos participantes e controle da frequência pode contribuir para a estimativa das metas e resultados pretendidos ${ }^{21}$. Consequentemente, o que se espera no planejamento em saúde para a promoção de saúde é melhorar a operacionalização e avaliação das ações, para assim serem aperfeiçoadas a fim de verificar o impacto da viabilidade econômica ${ }^{21,22}$.

A falta de clareza e de ferramentas que possibilitem um planejamento adequado das ações pode resultar em menores níveis de sucesso no contexto do $\mathrm{PAS}^{8}$. O planejamento não garante $100 \%$ de sucesso da ação, mas permite ao gestor a sua intencionalidade, além de ajudar a desenvolver as ações e serviços e otimizar os recursos disponíveis (humanos, estruturais, organizacionais, financeiros e materiais) ${ }^{8}$. Deste modo, fica evidente a necessidade de pesquisadores aproximarem as evidências científicas para os profissionais ${ }^{23}$, para assim reforçar entre os profissionais de saúde a importância de um grupo gestor para o planejamento em saúde, conforme as peculiaridades da territorialidade. Além de fomentar mais cursos para capacitação em planejamento e avalição das ações para as $\mathrm{PC} / \mathrm{AF}^{12}$.

$\mathrm{O}$ estudo tem como pontos fortes a análise das propostas de ações de $\mathrm{PC} / \mathrm{AF}$ pelos profissionais de educação física atuantes na APS, na qual contemplam também os profissionais atuantes no PAS. Isso permitiu compreender de que forma ocorre o planejamento, a operacionalização e a estrutura do processo para implementação das ações de $\mathrm{PC} / \mathrm{AF}$ no território nacional. Enquanto limitações, é importante considerar que as propostas analisadas foram decorrentes de trabalhos destinados à conclusão do Curso de Aperfeiçoamento do PAS, não sendo possível avaliar a viabilidade da implementação das propostas. Nesse sentido, apesar das propostas serem elaboradas de forma factível aos contextos de trabalho dos profissionais, desconhece-se se foram implementadas ou se houve adequação dos elementos e metodologias previamente propostos. No entanto, estudos estão sendo elaborados para dar continuidade a este acompanhamento.

Assim, o estudo mostra que as propostas, em sua maioria, ofereceram poucas informações sobre metodologias, instrumentos, cronogramas de execução, recursos profissionais e financeiros necessários. Além disso, não contemplam informações importantes sobre o público-alvo atendido e o diagnóstico situacional em saúde.
Avalia-se a necessidade de fornecimento de aprofundamento nos conhecimentos teóricos, técnicos e procedimentais no planejamento em saúde para as ações a serem implementadas na APS. Dessa forma, será possível conhecer as estratégias de alcance e impacto na população, adoção e sustentabilidade das ações pelas equipes de saúde e manutenção dos resultados esperados.

\section{Conflito de interesse}

Os autores declaram não haver conflito de interesse.

\section{Financiamento}

Curso de aperfeiçoamento (2015-2017) - Implementação da Política Nacional de Promoção da Saúde: Programa Academia da Saúde. Financiamento Diretoria Executiva do Fundo Nacional de Saúde - Auxílio financeiro. Chamada Pública 2014 - Iniciativas Educacionais Aplicadas à Vigilância em Saúde.

\section{Contribuição dos autores}

Todos os autores contribuíram para o desenvolvimento do manuscrito. Manta SW, concepção de delineamento do estudo, redação, análise estatística, análise dos dados e revisão crítica relevante do conteúdo intelectual; Sandreschi $\mathrm{PF}$, redação, análise dos dados, revisão crítica relevante do conteúdo intelectual. Quadros EM, análise documental e redação. Souza PV, análise documental e redação. Rech CR, Benedetti TRB, concepção da proposta em edital com financiamento, implantação do curso, redação e revisão crítica do conteúdo intelectual. Todos os autores aprovaram a versão final do manuscrito a ser publicado.

\section{Referências}

1. Brasil. Política Nacional de Promoção da Saúde- PNPS: revisão da Portaria MS/GM nº 687, de 30 de março de 2006/ Ministério da Saúde, Secretaria de Vigilância em Saúde, Secretaria de Atenção à Saúde. - Brasília: Ministério da Saúde, 2015.

2. Malta D, Silva Júnior JB. O plano de ações estratégicas para o enfrentamento das doenças crônicas não transmissíveis no Brasil e a definição das metas globais para o enfrentamento dessas doenças até 2025: uma revisão. Epidemiol Serv Saúde. 2013;22(1):151-64.

3. Brasil. Plano de ações estratégicas para o enfrentamento das doenças crônicas não transmissíveis (DCNT) no Brasil 2011-2022. Ministério da Saúde. Secretaria de Vigilância em Saúde. Departamento de Análise de Situação de Saúde. Brasília: Ministério da Saúde, 2011.

4. Wolker SM, Sandreschi PF, Tomicki C, Konrad LM, Quadros EN, Ribeiro CG, et al. Monitoramento do programa academia da saúde de 2015 a 2017. Rev Andal Med Deporte. 2020;13(1):16-20. 
5. Sá GBAR, Dornelles GC, Cruz KG, Amorim RCA, Andrade SSCA, Oliveira TP, et al. O Programa Academia da Saúde como estratégia de promoção da saúde e modos de vida saudáveis: cenário nacional de implementação. Ciênc Saúde Coletiva. 2016;21(6):1849-59.

6. Azevedo J, Mundstock E. Efeitos da prática orientada de exercícios físicos em pacientes do SUS no município de Canela, Brasil. Rev Bras Ciênc Esporte. 2018;40(4):339-45.

7. Melo VMC, Lemos EC, Marins AM, Silva BCR, Albuquerque AMT, Aros LJL, Tassitano RM. Performance of Physical education Professionals from the academia da cidade Program in Primary Health care in recife. Rev Bras Ativ Fís Saúde 2016;21(5):483-93.

8. Brasil. Ministério da Saúde. Secretaria de Atenção Primária à Saúde. Departamento de Promoção da Saúde. Programa Academia da Saúde: caderno técnico de apoio a implantação e implementação / Ministério da Saúde, Secretaria de Atenção Primária à Saúde, Departamento de Promoção da Saúde. - Brasília: Ministério da Saúde, 2019. 220 p.

9. Guarda FRB, Silva RN, Feitosa WMN, Farias JM, Neto PMS, Araújo Junior JLAC. Autopercepção acerca do objetivo, objeto e produtos do trabalho dos Profissionais de Educação Física que atuam no Programa Academia da Saúde. Rev Bras Ativ Fís Saúde. 2016;21(5):400-9.

10. Guarda FRB, Silva RN, Feitosa WMN, Neto PMS, Araújo Junior JLAC. Caracterização das equipes do Programa Academia da Saúde e do seu processo de trabalho. Rev Bras Ativ Fís Saúde. 2015;20(6):638-40.

11. Freitas EF, Moreira OC, Oliveira CEP, Doimo LA, Loch MR. Prevalência de diabetes mellitus e prática de exercício em indivíduos que procuraram atendimento na estratégia saúde da família de Viçosa/MG. Rev Educ Fís. 2015;26(4):549-56.

12. Brasil. Curso de aperfeiçoamento em implementação da Política Nacional de Promoção da Saúde: Programa Academia da Saúde. Ministério da Saúde, Universidade Federal de Santa Catarina. - 2. ed. - Brasília: Ministério da Saúde, 2017.

13. Gil AC. Como elaborar projetos de pesquisa. $4^{\mathrm{a}}$ ed. São Paulo: Atlas, 2002.

14. Silva RN, Guarda FRB, Hallal PC, Martelli PJL. Avaliabilidade do Programa Academia da Saúde no Município do Recife, Pernambuco, Brasil. Cad Saúde Pública.2017;33(4):e00159415.
15. Amorim TC, Knuth AG, Cruz DKA, Malta DC, Reis RS, Hallal PC. Descrição dos programas municipais de promoção da atividade física financiados pelo Ministério da Saúde. Rev Bras Ativ Fis Saude. 2013;18(1):63-74.

16. Florindo AA, Nakamura PM, Farias Junior JC, Siqueira FV, Reis RS, Cruz DKA, et al. Promoção da atividade física e da alimentação saudável e a saúde da família em municípios com academia da saúde. Rev Bras Educ Fís Esporte. 2016;30(4):913-24.

17. Harvey G, Kitson A. PARIHS revisited: from heuristic to integrated framework for the successful implementation of knowledge into practice. Implementation Science. 2016;11(33):1-13.

18. Rycroft-Malone J, Seers K, Eldh AC, Cox K, Crichton N, Harvey G, et al. A realist process evaluation within the Facilitating implementation of Research Evidence (FIRE) cluster randomized controlled international trial: an exemplar. Implementation Science. 2018;13(138):1-15.

19. Guarda FRB, Silva RN, Araújo Junior JLAC, Freitas MIF, Neto PMS. Intervenção do profissional de educação física: formação, perfil e competências para atuar no Programa Academia da Saúde. Rev Pan-Amaz Saude. 2014;5(4):63-74.

20. Cranley LA, Keefe JM, Taylor D, Thompson G, Beacom $\mathrm{AM}$, Squires JE, et al. Understanding professional advice networks in long-term care: an outside-inside view of best practice pathways for diffusion. Implementation Science. 2019;14(10):1-13.

21. Estabrooks PA, Wilson KE, McGuire TJ, Harden SM, Ramalingam N, Schoepke L, et al. A Quasi-experiment to assess the impact of a scalable, community-based weight loss program: combining reach, effectiveness, and cost. J Gen Intern Med 2017;32(Suppl 1):24-31.

22. Sun Y, You W, Almeida F, Estabrooks P, Davy B. The Effectiveness and cost of lifestyle interventions including nutrition education for diabetes prevention: a systematic review and meta-analysis. J Acad NutrDiet 2017;117(3):1-54.

23. Becker LA, Rech CR, Reis RS. Evidence-based public health: concepts, principles and applications to promote physical activity in the Brazilian context. Rev Bras Cineantropom Desempenho Hum. 2019;21:e56303.

Recebido: 25/06/2020

Aprovado: 20/11/2020

\section{Como citar este artigo:}

Manta SW, Sandreschi PF, Quadros EN, Souza PV, Rech CR, Benedetti TRB. Planejamento em saúde sobre práticas corporais e atividade física no Programa Academia da Saúde. Rev Bras Ativ Fís Saúde. 2020;25:e0168. DOI: 10.12820/rbafs.25e0168 